\title{
Folie a deux
}

Pratikshya Chalise, ${ }^{1}$ Sandip Subedi, ${ }^{2}$ Pawan Sharma'

'Transcultural Psychosocial Organization,Kathmandu, Nepal, ${ }^{2}$ Universal College of Medical Sciences, Bhairahawa, Nepal.

\begin{abstract}
Shared psychotic disorder is a rare psychiatric disorder. It is defined when a primary psychotic person imposes his/her delusional beliefs to the other person usually in a close relationship. Occurrence of this disorder among family members and close friends has been described. However, its exact incidence and prevalence is not known. Since such case has not been reported in Nepal to best of our knowledge, we present a case of shared delusional disorder along with brief review of literature. This case report describes a case of common shared persecutory delusion in husband and wife.
\end{abstract}

Keywords: folie a deux; shared psychotic disorder.

\section{INTRODUCTION}

Shared psychotic disorder also known as Folie à deux was first described in 1877 by Laségue and Falret in France. ${ }^{1}$ The essential feature of this disorder is a delusion that develops in an individual who is involved in a close relationship with another person who already has a psychotic disorder (the primary case). The secondary case is frequently less

intelligent, more gullible, more passive, or more lacking in self-esteem than the primary case. ${ }^{2}$ It is found between siblings, spouse, parent-offspring and even close friends. Rarely all of the family members may be involved. ${ }^{3}$ The etiology for the development of folie a deux is exactly not known however genetic susceptibility and family history are considered strong factors. Other factors include female gender, mental retardation, histrionic behavior, social isolation, life events. Multiple modality of treatment are used in this disorder like separation, antipsychotics, individual and group psychotherapy, and family therapy. ${ }^{4}$ Many years after its first description, folie á deux remains an interesting and challenging disorder to psychiatrists, especially concerning its psychopathology and treatment. We report a similar case in the rural setting of Nepal and its management in the tertiary care centre.

\section{CASE REPORT}

A female patient and her husband was brought to UCMS Psychiatry OPD by their elder daughter with the following details.

\section{Case A (Wife- the primary case):}

A 39 year old housewife of Hindu nuclear family from Nawalparasi district of Nepal with primary level education, no past or family history of psychiatric illness presented with acute and progressive illness of three weeks characterized by firm belief that her neighbors talked about her behind her back. She stopped going to her neighbors' home and didn't interact with them at all claiming that they were making a plot to harm her and her family. Frequent visit to the faith healers firmed her beliefs. Her predominant mood during the course of illness was fearful. As her condition deteriorated with symptoms like lack of sleep, screaming at night, abusing the neighbors and violent behaviour against them she was brought to our tertiary centre for admission. . There was no history of substance abuse, head injury or any chronic physical illness.

Her general physical examination and systemic 
examination were within normal limits. On mental status examination during the time of admission, patient had delusion of reference and persecution, her predominated mood was anxious. Her routine investigations were within normal limits.

\section{Case B (the husband- secondary case or recipient)}

The husband of the patient, a 40 year man who studied up to secondary level presented at the same time with the symptoms of acute onset characterized by decreased sleep and suspiciousness. The husband too started to believe in his wife's belief that their neighbors are against him/his family and wanted to kill them. He frequently quarreled with the villagers, accused them and resisted any social calls made by them. One day prior to the admission patient physically abused his neighbor following which he was brought to the hospital. There was no significant past or family history (maternal or paternal side) and no history of substance abuse, head injury or chronic physical illness.

General physical and systemic examination revealed no abnormality. Mental state examination on the day of admission revealed restless, agitated behavior with anxious mood and presence of delusion of persecution. Preliminary laboratory investigations were with in normal limits.

On psycho diagnostic assessment, Thematic Apperception Test and Rorschach Ink Blot Test indicated psychotic anxiety, less capacity to tolerate stress and cognitive load. There were some bizarre responses and some responses indicated low self-esteem.

The patients were admitted in female and male psychiatry ward respectively, separated from each other. The wife was started with olanzapine $5 \mathrm{mg}$ per day and increased gradually up to $20 \mathrm{mg}$ per day and lorazepam was also given for decreased sleep and agitation which was gradually tapered off. She was improved gradually and reached partial remission but was discharged on request. During first follow up in OPD improvement was noted with resolution of psychotic symptoms. On her subsequent follow up she reached her premorbid state. The husband was started with Olanzapine $5 \mathrm{mg} /$ day, increased to $10 \mathrm{mg} /$ day and lorazepam $1 \mathrm{mg}$ per day. He was also separated from his wife for few days. He showed marked improvement after few days of admission reached complete remission in around a week suggesting that separation might have worked faster than the antipsychotic medication. So the diagnosis of induced delusional disorder (folie à deux) as per ICD 10 was made. He persisted to show complete remissions in his subsequent follow ups as well even after stopping Olanzapine.

\section{DISCUSSION}

After a complete clinical assessment, psychological testing and mental status examination we diagnosed the index case as folie a deux as the husband had same delusion as wife, his symptoms had started after the symptoms of his wife and he showed complete improvement after being separated from his wife and few days of antipsychotic therapy.. According to Gralnick shared delusional disorder comprises of four types: Folie impose, folie simultanée, folie communiqué, folieinduite. ${ }^{5}$ Our case is in concordance with the description of folie impose in which the dominant individual usually suffering from a psychotic illness, imposes his/her delusion on the secondary; more subdued individual.

The literature shows that males and females are affected with equal frequency and there is equal prevalence in younger and older patients. The majority of the cases seen are within the nuclear family, commonly involving siblings, spouse or parent-off springs. Similarly it might also occur within friends i.e. genetically unrelated individuals. Social isolation has often been described as a major risk factor for the development of folie à deux. $A$ number of other factors reported in the secondary cases are: passive personality, cognitive impairment, language difficulties, and life events. ${ }^{6}$ Most studies explaining the psychopathology of this disorder have mentioned the role of genetic susceptibility, but occurrence of the disorder in friends and spouse also suggest an additional environmental factor as exogenous source. ${ }^{7}$

There is no general consensus for systematic treatment regimen for this disorder. Inconsistencies in literature regarding treatment modalities exist between either one of two; separation as the sole treatment for the secondary, or psychotherapy and medical intervention in conjunction with separation, for cure of the secondary. ${ }^{4}$ On the contrary; case with fatal outcome after separation have been reported suggesting separation might increase risk of adverse outcomes. ${ }^{8}$ However the primary in our case was treated with antipsychotics and was continued in the follow up and the secondary was treated with antipsychotic and tapered off within one month of treatment. No relapse of symptoms have occurred in the six month follow up highlighting separation played a vital roe in symptom remission in the secondary. Further research is warranted in this field in understanding the psychopathology, biological aspects and management protocol. 


\section{REFERENCES}

1. La folie à deux ou folie communiquée (FALRET \& LASÈGUE, 1877) [Internet]. [cited 2015 Dec 29]. Available from: http:// asklepios.chez.com/falret/folie.htm

2. Al-Huthail YR. Shared psychotic disorder. Neurosci Riyadh Saudi Arab. 2002 Oct;7(4):304-6

3. Jolfaei AG, Isfahani MN, Bidaki R. Folie à deux and delusional disorder by proxy in a family. J Res Med Sci Off J Isfahan Univ Med Sci. 2011 Mar;16(Suppl1):S453-5.

4. Arnone D, Patel A, Tan GM-Y. The nosological significance of Folie à Deux: a review of the literature. Ann Gen Psychiatry. 2006 Aug 8;5:11.
5. Gralnick A. Folie a deux-the psychosis of association. Psychiatr Q. 2014 Jan 15;16(3):491-520.

6. Silveira JM, Seeman MV. Shared psychotic disorder: a critical review of the literature. Can J Psychiatry Rev Can Psychiatr. 1995 Sep;40(7):389-95.

7. Mouchet-Mages S, Gourevitch R, Lôo H. [Folie à deux: update of an old concept regarding two cases]. L'Encéphale. 2008 Jan;34(1):31-7.

8. Talamo A, Vento A, Savoja V, Di Cosimo D, Lazanio S, Kotzalidis GD, et al. Folie à deux: double case-report of shared delusions with a fatal outcome. Clin Ter. 2011;162(1):45-9. 